\title{
openheart Lower coronary plaque burden in patients with HIV presenting with acute coronary syndrome
}

\author{
E J O'Dwyer, ${ }^{1,2,3}$ P Bhamra-Ariza, ${ }^{1,4}$ S Rao, ${ }^{1,3}$ S Emmanuel, ${ }^{1,5}$ A Carr, ${ }^{1,2}$ \\ C J Holloway ${ }^{1,2,3,4}$
}

To cite: O'Dwyer EJ, BhamraAriza P, Rao S, et al. Lower coronary plaque burden in patients with HIV presenting with acute coronary syndrome. Open Heart 2016;3:e000511. doi:10.1136/openhrt-2016000511

Received 28 July 2016 Revised 20 October 2016 Accepted 17 November 2016

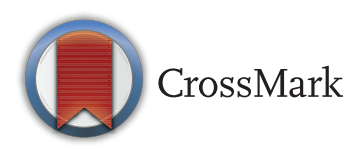

\footnotetext{
${ }^{1}$ St. Vincent's Hospital Darlinghurst and the University of New South Wales, Sydney, New South Wales, Australia

${ }^{2}$ University of New South Wales, Sydney, New South Wales, Australia

${ }^{3}$ Victor Chang Cardiac

Research Institute, Darlinghurst, New South Wales, Australia

${ }^{4}$ Frimley Health NHS Foundation, Surrey, UK ${ }^{5}$ University of Notre Dame, Sydney, New South Wales, Australia
}

Correspondence to Dr EJ O'Dwyer; eoinjude@gmail.com

\section{ABSTRACT}

Objective: Treated HIV infection is associated with a higher incidence of coronary artery disease and myocardial infarction, although the mechanisms remain unclear. We sought to characterise the burden of coronary artery disease in men with HIV using retrospective data from invasive coronary angiograms in patients presenting with acute coronary syndrome (ACS).

Methods: Demographic and coronary angiographic data were obtained from 160 men with ST elevation myocardial infarction, non-STEMI or high-risk chest pain; 73 HIV-infected cases and 87 age-matched controls. The burden of coronary disease was calculated using the Gensini Angiographic Scoring System by 2 independent cardiologists blinded to HIV status.

Results: The 2 groups were matched for age, sex and cardiac event subtype and there was no difference in rates of smoking or cholesterol levels. Compared with control participants, patients with HIV had higher usage of antihypertensives (46 (63\%) vs $30(35 \%)$, $p<0.001)$ and statins (47 (64\%) vs $29(33 \%)$, $\mathrm{p}<0.001)$. There was no difference in plaque distribution between both groups; however, the Gensini score was $42 \%$ lower in cases with HIV than in controls $(p<0.03)$. $C$ reactive protein was higher in cases with HIV $(13.4 \pm 15.4$ vs $3.7 \pm 3.6)$.

Conclusions: Men with HIV presenting with ACS paradoxically had a lower burden of coronary plaque than matched controls, despite more aggressive risk factor management, suggesting that plaque vulnerability, rather than total burden of atherosclerosis, may be important in the pathophysiology of coronary artery disease in men with HIV.

\section{INTRODUCTION}

Since the advent of antiretroviral therapy (ART), there has been a marked reduction in mortality associated with HIV. ${ }^{1}$ In patients with treated HIV, accelerated cardiovascular disease is now emerging as a major contributor to morbidity, including a higher incidence of acute myocardial infarction and sudden cardiac death. ${ }^{2}$ The WHO predicts

\section{KEY QUESTIONS}

What is already known about this subject?

- The HIV pandemic has been a major global public health issue with a prevalence of 35 million. With the advent of antiretroviral therapy (ART), HIV is now a chronic treatable disease, though it is associated with accelerated cardiovascular disease, including increased coronary vascular events and myocardial dysfunction.

\section{What does this study add?}

- This study using invasive coronary angiography to measure disease burden in patients with HIV. In HIV-positive participants presenting with acute coronary syndrome (ACS), our study reveals a paradoxically lower burden of coronary plaque. We suggest that chronic inflammation causes plaque vulnerability rather than total burden of atherosclerosis is a mechanism of ACS in patients with HIV.

How might this impact on clinical practice?

- Given the recent NEJM publication (2015; 373: 795-807) which demonstrated improved mortality and morbidity with early initiation of ART at diagnosis, we believe that our study offers further support that early treatment may prevent long-term cardiac complications of HIV infection, as outlined by the new treatment model endorsed by the WHO.

that by 2030 both HIV/AIDS and ischaemic heart disease (IHD) will be among the top three causes of global mortality and disability-adjusted day-years. ${ }^{3}$ While the incidence of HIV-related cardiovascular disease is declining, ${ }^{4}$ the interplay between both diseases remains of global importance.

The aetiology of IHD in patients with HIV is likely to be multifactorial and the pathophysiology remains unclear. Direct viral factors, delay in initiation of ART and the use of subclasses of $\mathrm{ART}^{4}$ may play a role in the development of coronary artery disease (CAD) in patients living with HIV. The 
inflammatory response to infection, in particular, with raised levels of inflammatory mediators, in well-treated participants with HIV, may play a casual role in the increased acute coronary syndrome (ACS) rate. ${ }^{5}$ In keeping with a novel pathogenesis, observational studies have shown cardiac events with a fresh thrombus but no overt atherosclerotic lesions ${ }^{6} 7$ CT coronary angiography (CT-CA) studies have identified increased prevalence of high-risk plaque, as well as increased plaque burden in groups of asymptomatic participants with $\mathrm{HIV} ;{ }^{8-10}$ however, there are limited invasive studies examining the association between ACS and plaque burden in patients with HIV. ${ }^{11}$

We quantified the burden of coronary disease in men with HIV using retrospective data from invasive coronary angiograms in patients presenting for investigation of ACS. We hypothesised that infection with HIV may be associated with differences in plaque distribution and overall burden, reflecting a distinct form of vasculopathy, compared with negative matched controls.

\section{METHODS}

\section{Study design}

Demographic and invasive coronary angiogram data were retrospectively collected from the St Vincent's Hospital clinical database regarding men with ACS defined as per American Heart Association as either ST elevation myocardial infarction (STEMI) or non-STEMI (NSTEMI) or high-risk chest pain/unstable angina. Approval for the study was granted by the Human Research and Ethics Committee (HREC) LNR/15/ SVH/45 of St Vincent's Hospital, Sydney.

Data from 73 participants with HIV and 87 controls presenting between January 2005 and March 2014 were obtained from St Vincent's Hospital, Sydney, Australia (figure 1).

\section{Participants}

Cases were men, $>18$ years of age with serologically confirmed HIV infection. Controls were matched for age, sex and smoking status from the hospital's interventional angiography database. Controls were checked against the NSW HIV reference laboratory for history of HIV infection. We excluded women because of the limited number of data available, as well as patients with endstage renal disease (defined as an estimated glomerular filtration rate $<30 \mathrm{~mL} / \mathrm{min} / 1.73 \mathrm{~m}^{2}$ ) or liver disease (defined as documented cirrhosis or known liver impairment as documented on the day of admission).

\section{Data collection}

Data on comorbidities including cardiac risk factors, medication history and laboratory results were obtained through individual record review as documented on the day of admission with ACS.

$\mathrm{C}$ reactive protein (CRP) was measured using an immunoturbidimetric assay on an ADVIA 2400 general
Study Design

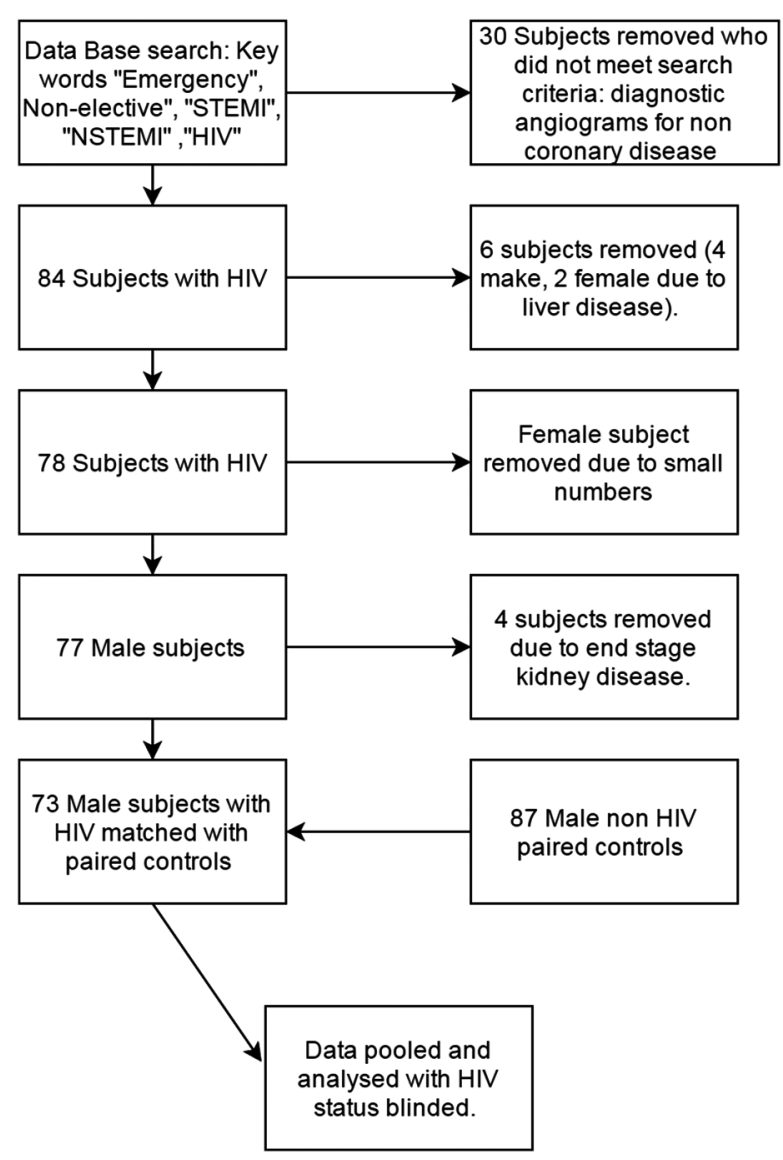

Figure 1 Study design.

chemistry analyser (Siemens Healthcare Diagnostics, Frimley, UK) applied using the manufacturer's recommendations. Presenting cardiac diagnosis was retrieved as documented by admitting physician as the indication for invasive coronary angiogram. ACS was defined as STEMI, NSTEMI or unstable angina requiring cardiac angiography, as determined by the treating cardiologist.

\section{Plaque burden quantification using Gensini scoring}

All coronary angiograms were analysed independently by two cardiologists who were not involved in the patient's medical care (PB-A, SR). The contrast-filled catheter was used for image magnification calibration. Diameters of reference and stenotic coronary arteries were measured by computerised quantitative methods using the Quantcor Coronary Analysis software (Siemens Medical Solutions, Erlangen, Germany; figure 2A, B).

The severity of CAD was scored using the Gensini score. ${ }^{12}$ According to the angiographic stenosis, each narrowing between $0 \%$ and $25 \%, 25 \%$ and $50 \%, 50 \%$ and $75 \%, 75 \%$ and $90 \%, 90 \%$ and $99 \%$, and $100 \%$ was scored as 1, 2, 4, 8, 16 or 32 points, respectively. As 

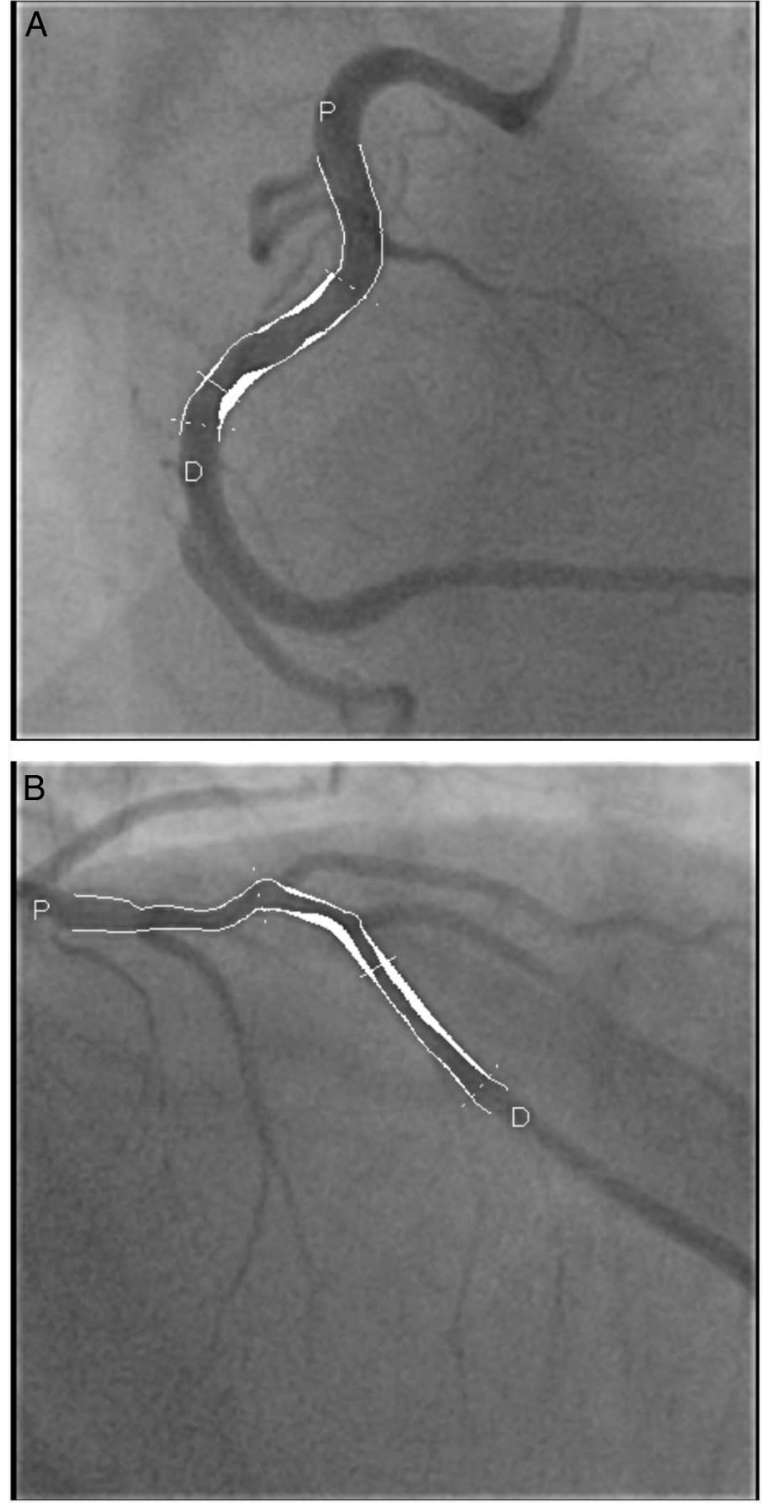

Figure 2 (A) Quantitative plaque analysis of the RCA. (B) Quantitative plaque analysis of the LAD. Each stenosis is given a score and multiplied by a factor depending on the location of the stenosis in the coronary tree in order to calculate the Gensini score. LAD, left anterior descending artery; $\mathrm{RCA}$, right coronary artery.

previously described, ${ }^{12}$ each score was then multiplied by a factor that represents the significance of the lesion's location in the coronary artery tree: a factor of 5 for the left main lesion; 2.5 for the proximal left anterior descending (LAD) or left circumflex (LCX) artery; 1.5 for the mid-segment LAD and LCX; 1 for the distal segment of LAD and LCX, first diagonal branch, first obtuse marginal branch, right coronary artery, posterior descending artery and intermediate artery; and 0.5 for the second diagonal and second obtuse marginal branches. The Gensini score was expressed as the sum of the scores for all three coronary arteries to evaluate the entire extent of $\mathrm{CAD}$, with higher scores indicating a greater disease burden.

\section{Statistical analysis}

Normality of data was tested using the Kolmogorov-Smirnov test. Continuous variables are presented as mean $\pm \mathrm{SD}$ and discrete variables are presented as numbers (percentages). Fisher's exact test was used to compare dichotomous data. The unpaired Student's t-test (when normally distributed) or Mann-Whitney U test (for non-parametric data) was used to compare continuous variables between participants with HIV infection and controls, as appropriate. Segmental data were averaged on a per-participant basis to control for clustering of segments within each participant. Bivariate correlations were assessed using the Pearson ' $R$ ' or Spearman ' $R_{S}$ ' coefficient, as appropriate. Linear regression analysis was used to examine associations with the Gensini score and other variables. All statistical tests were twotailed and a $p$ value of $<0.05$ was considered significant. All analyses were performed using SPSS V.20 (IBM, Armonk, New York, USA).

\section{RESULTS}

\section{Participant characteristics}

HIV-infected cases and controls were well matched for age, sex and smoking status, and had similar coronary events and lipid levels (table 1). Relative to controls, the cases had higher triglyceride levels $(2.5 \pm 1.7$ vs 1.8 $\pm 1.5 \mathrm{mmol} / \mathrm{L}, \mathrm{p}=0.04$ ), were more likely to have a history of medically treated hypertension $(63 \%$ vs $35 \%, \mathrm{p}=0.001)$ and were twice as likely to be on statin therapy at admission $(p<0.001)$. Cases also had a lower prevalence of type 2 diabetes mellitus (28\% vs $13 \% \mathrm{p}=0.02)$ and lower body mass index (BMI; $24.8 \pm 4.3$ vs $29 \pm 7.4 \mathrm{~kg} / \mathrm{m}^{2}, \mathrm{p}=0.001$ ).

\section{Participants with HIV}

Mean duration of HIV infection was $11 \pm 4$ years to the date of angiography. All but two cases were receiving ART, and $65(89 \%)$ had an undetectable HIV viral load. Of the participants with HIV on ART, 21 (29\%) were on abacavir and $50(71 \%)$ were receiving a HIV protease inhibitor.

After removing extreme outliers, CRP values were available for $35(48 \%)$ participants from the HIV group and $36(41 \%)$ from the control group. CRP values were significantly higher in patients with HIV compared with controls $(13.4 \pm 15.4$ vs $3.7 \pm 3.6, \mathrm{p}<0.001)$. When cases with detectable plasma HIV were excluded from the analysis, CRP continued to be higher in the HIV group compared with controls $(12.6 \pm 15$ vs $3.7 \pm 3.6, \mathrm{p}=0.03)$.

\section{Severity of coronary disease on angiography}

When comparing the total number of diseased coronary vessels, there was no difference between participants with HIV and controls $(1.94 \pm 1.5$ vs $2.3 \pm 1.3, \mathrm{p}=0.15)$. After analysis, there was no difference in the distribution of plaque between both groups $(p=0.15)$. However, when the total burden of coronary disease was measured using the Gensini score, participants with HIV had a $38 \%$ lower mean total Gensini score compared with 
Table 1 Baseline characteristics of the study participants

\begin{tabular}{|c|c|c|c|}
\hline & Control participants $\mathrm{N}=87$ & HIV-positive participants $\mathrm{N}=73$ & p Value \\
\hline Age & $57 \pm 8$ & $55 \pm 8$ & 0.15 \\
\hline Male $(N)$ & 87 & 73 & \\
\hline Smoking n (\%) & $21(24)$ & $22(30)$ & 0.36 \\
\hline Diabetes n (\%) & $24(28)$ & $9(13)$ & 0.02 \\
\hline Triglycerides (mmol/L) & $1.8 \pm 1.5$ & $2.5 \pm 1.7$ & 0.04 \\
\hline Total cholesterol (mmol/L) & $4.7 \pm 1.3$ & $5 \pm 1.3$ & 0.14 \\
\hline LDL cholesterol (mmol/L) & $2.6 \pm 1.5$ & $2.9 \pm 1$ & 0.36 \\
\hline HDL cholesterol (mmol/L) & $1.3 \pm 0.3$ & $1.1 \pm 0.3$ & 0.22 \\
\hline BMI $\left(\mathrm{kg} / \mathrm{m}^{2}\right)$ & $29.0 \pm 7.4$ & $24.8 \pm 4.3$ & $<0.001$ \\
\hline STEMI n (\%) & $13(15)$ & $8(11)$ & 0.48 \\
\hline NSTEMI $\mathrm{n}(\%)$ & $41(47)$ & $30(41)$ & 0.48 \\
\hline High-risk chest pain n (\%) & 33 (38) & $35(48)$ & 0.20 \\
\hline Hypertension treatment $\mathrm{n}(\%)$ & $30(35)$ & $46(63)$ & $<0.001$ \\
\hline Statin usage $\mathrm{n}(\%)$ & 29 (33) & $47(64)$ & $<0.001$ \\
\hline Duration of HIV diagnosis (years) & NA & $11.02 \pm 4$ & NA \\
\hline Viral load $<50(\%)$ & NA & 89 & NA \\
\hline CD4 count & NA & $510 \pm 220$ & NA \\
\hline $\operatorname{CRP}(\mathrm{mg} / \mathrm{L})^{*}$ & $3.7 \pm 3.6$ & $13.4 \pm 15.4$ & $<0.001$ \\
\hline PIn (\%) & NA & (51) 70 & NA \\
\hline NRTI n (\%) & NA & (62) 85 & NA \\
\hline NNRTI n (\%) & NA & (68) 82 & NA \\
\hline Abacavir usage $\mathrm{n}(\%) \dagger$ & NA & $21(29)$ & NA \\
\hline
\end{tabular}

Continuous data are mean \pm SD unless otherwise indicated.

${ }^{*}$ As measured on the day of admission.

†As documented as part of ART regime on the day of admission.

BMI, body mass index; CRP, C reactive protein; HDL, high-density lipoprotein; LDL, low-density lipoprotein; NA, not available; NNRTI, non-nucleoside reverse transcription inhibitor; NRTI, nucleoside reverse transcription inhibitor; NSTEMI, non-ST elevation myocardial infarction; PI, protease inhibitor; STEMI, ST elevation myocardial infarction; UAP, unstable angina pectoris.

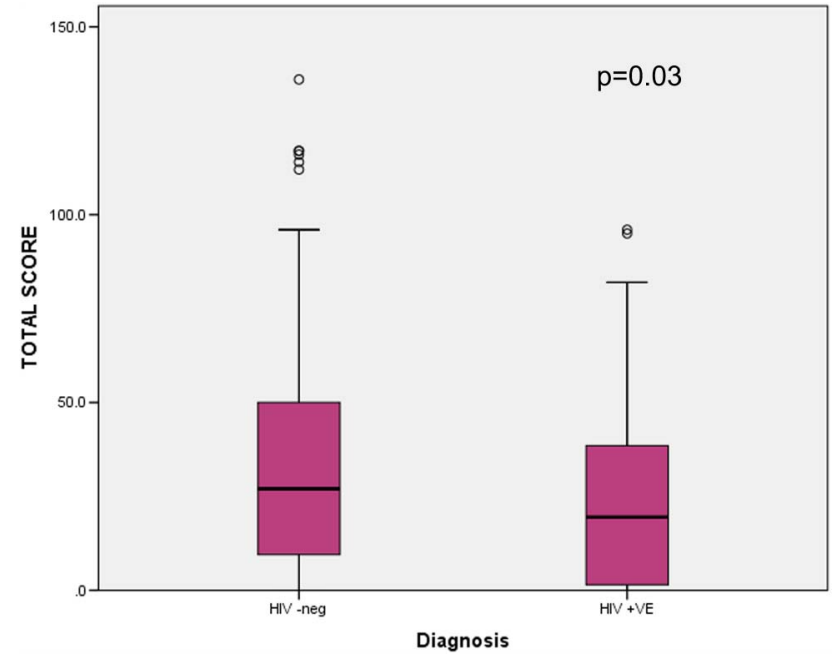

Figure 3 Comparative distributions of total Gensini scores expressed for participants with HIV versus controls.

controls (25.1 vs 36.5, mean difference 10, $\mathrm{p}=0.03$, figure 3), demonstrating a lower total atherosclerotic burden in patients with HIV. There was no difference in the Gensini score when comparing cases and controls with either STEMI/NSTEMI or unstable angina.

\section{Gensini score correlations}

On univariate analysis, there was an association between the total Gensini score and duration of known HIV infection ( $\mathrm{r}=0.30, \mathrm{p}=0.004)$ and high-density lipoprotein (HDL; $\mathrm{r}=0.276, \mathrm{p}=0.02$ ). When combining these two variables, and considering all risk factors as variables only in patients with HIV, there was a strong association between higher HDL levels and lower Gensini score $(\mathrm{r}=0.481, \mathrm{p}=0.005)$, suggesting a reduced total plaque burden in patients with higher HDL levels. There was no association between statin use and Gensini score $(\mathrm{p}=0.2)$.

A stepwise multiple linear regression analysis was employed to help determine which of the variables of interest (HIV status, age, STEMI/NSTEMI, total cholesterol, smoking, BMI, hypertension, diabetes, abacavir and statin usage) predicted a higher Gensini score. The multiple regression model identified HIV diagnosis as the most predictive factor for a lower Gensini score with a positive HIV diagnosis carrying a $\beta$ weight of $-20 \pm 6.9$ $(\mathrm{p}=0.004)$, which is in keeping with our finding that patients with HIV have a lower total plague burden.

\section{DISCUSSION}

There are limited coronary angiographic studies in patients with HIV presenting with ACS. In our study, we found a paradoxical lower burden of atherosclerotic plaque as assessed using the Gensini score in men with HIV than in controls, despite no difference in the plaque location or number of stenoses. Lower plaque burden was correlated with higher HDL cholesterol 
levels in patients with HIV. We propose that while there is a lower atherosclerotic burden, higher plaque vulnerability, possibly due to a chronic inflammatory process, may explain increased ischaemic events in the HIV population.

Previous studies using CT-CA have demonstrated a higher burden of coronary atherosclerotic plaque in asymptomatic patients with HIV and higher calcium scoring. ${ }^{9}{ }^{10}$ Furthermore, coronary plaque morphology is markedly different in participants with HIV, with asymptomatic participants showing increased incidence of low attenuation cholesterol plaque, positively remodelled plaque and high-risk plaque. ${ }^{9}$ Conversely, we found that HIV-infected men with ACS have a lower total plaque burden than age-matched controls. The finding of a lower total Gensini score does not appear to suggest a lower risk in the HIV group, but rather may represent the presence of plaque with high-risk features.

This mechanism of ACS in patients with HIV is not well established but is most likely multifactorial. There is a higher incidence of traditional risk factors in patients with HIV including abnormal lipid profiles and large population studies have shown a higher prevalence of smoking. ${ }^{13}{ }^{14}$ When these studies were adjusted for risk factors, a greater risk of cardiac events remains, which suggests that non-traditional risk factors are influencing outcome. We matched our patients for smoking status and age and, aside from triglycerides, there was no difference in lipid profiles between the groups. Previous studies have shown higher serum triglyceride levels in patients with HIV, which are thought to be due to the metabolic complications of ART. ${ }^{14}$ In our study, twice as many people with HIV on the day of admission were on a statin compared with controls. We suggest that this most likely represents more aggressive risk factor management by local physicians, though the duration of statin treatment is unknown. The lipid-lowering and antiinflammatory effects of statins are well established, but whether these translate to a lower risk of ACS in patients with HIV has not been established. Observational studies in patients with HIV have previously shown a reduction in inflammatory markers with statin usage; ${ }^{15}$ however, a recent study, though underpowered, suggests that statin usage results in a lower burden of unstable plaque while having no demonstrable effect on arterial inflammation. ${ }^{16}$ Randomised controlled trials are required to determine whether statins reduce the cardiovascular risk in the HIV population.

Previous studies ${ }^{17}$ have suggested a link between the use of abacavir and increased coronary events. We did not demonstrate a correlation between the usage of abacavir and plaque burden; however, it should be noted that historical usage is unknown.

Unique to the HIV population is the presence of chronic viraemia and subsequent inflammation. The SMART $^{17}$ study was pivotal in showing a link between viraemia and cardiovascular events in patients with HIV. Our patients were well treated and predominantly virally suppressed, though CRP remains elevated in keeping with a chronic inflammatory condition. Undetectable viral loads do not equate to a lack of ongoing inflammation, while inflammation and immune activation are reduced with treatment it does not normalise. ${ }^{18}$ We propose that a persistent inflammatory response may alter the architecture of atherosclerotic plaque and perhaps explain partially why there is a higher incidence of cardiovascular events in this group.

There is a global push towards initiation of ART at diagnosis of HIV. The START ${ }^{19}$ study demonstrated improved mortality and morbidity when initiating ART at an early stage; however, it did not demonstrate any benefit with regard to the incidence of cardiovascular events, possibly due to the relatively short follow-up of 3 years and the median duration of known HIV infection of 1 year. Compared with patients in the START study, our cohort had a 10 times longer exposure to HIV. The majority of patients in our study initiated ART when CD 4 counts fell below $<350$ as per local guidelines. It is unclear whether starting ART earlier would have decreased these cardiac events and affected cardiac end points.

Non-traditional risk factors such as inflammation and the recruitment of inflammatory cells and the upregulation of inflammatory cytokines may all have a role in unstable plaque formation and may partially explain our findings. The mechanism for persistent inflammation in the HIV population, whom are virally suppressed and well treated is uncertain. A recent review on immune activation and cardiovascular disease describes how monocyte-mediated/macrophage-mediated inflammation and injury in atherosclerotic lesions are increased in HIV infection despite treatment. ${ }^{20} \mathrm{HIV}$ is also associated with abnormal functioning of monocytes including impaired cholesterol efflux by foam cells and procoagulant effects, that is, monocytes from HIV patients are more likely to become foam cells, leading to higher CVD risk. The review also describes numerous ongoing trials looking at downregulating immune activation in HIV patients with both chemotherapies and newer biological agents. ${ }^{20}$

Recent animal studies have shown persistent upregulation of cytokines and damage to the myocardium despite viral suppression in HIV. These cytokines and chemoreceptors are now targets for new antiviral therapies (CCR5 inhibitors) and have shown improvement in cardiac function, when downregulated, in animal studies. ${ }^{21}$ With regard to inflammation, HIV behaves similarly to other chronic conditions and has been associated with higher inflammatory markers. Zanni et af have shown that immune activation plays a role by showing marked arterial inflammation with cardiac fluorodeoxyglucose-positron emission tomography scanning and associated high levels of the monocyte activation marker sCD16, interleukin-6 and CRP in participants with HIV without overt symptoms of cardiovascular disease. This inflammation is more apparent in 
the early non-treated stages of the infection but persists to a lesser extent with viral suppression. ${ }^{9}$

As an independent observation within the HIV group, the participants with the lowest Gensini scores had higher serum HDL. HDL is associated with improved cardiac outcomes in the non-HIV population and, prior to the usage of ART, a falling HDL level was a poor prognostic sign and was associated with rising low-density lipoprotein levels and viraemia. It is unclear if statin usage is a factor in this correlation in our study and whether HDL levels within the HIV group affected outcome. Further studies are required to assess the effect of statin usage on cardiac outcomes in patients with HIV.

Our study has several limitations, including a modest sample size, inclusion of men only and retrospective design. We have no end point clinical data on mortality. There was insufficient detailed information of illicit drug use available in the patients' notes to exclude this as a confounding factor. Medication history was retrieved as documented on the day of admission, so previous ART therapy could not be recorded. Duration of usage and previous usage of other medications both cardiac and ART were not obtained. Some pathology results were not available on all patients. Although coronary stenosis severity, as assessed by invasive angiography, is associated with clinical outcomes, its ability to look beyond a simple measure of 'luminal narrowing' is limited; it is known that some culprit lesions responsible for AMI may appear mild on angiography. ${ }^{22}$ The amount of plaque may be underestimated when using angiography, as a result of expansive or positive vessel remodelling. ${ }^{23}$ Our study did not use intracoronary imaging, which has been shown to potentially identify different plaque morphologies. However, the Gensini score has been strongly correlated with plaque burden as assessed with Intravascular ultrasound (IVUS) and therefore may be used to provide valid assessments of CAD burden. ${ }^{24}$

In this population, HIV-infected men with ACS had lower total atherosclerotic plaque than controls. This paradoxical finding of reduced plaque burden, in a group of higher risk patients, who are generally known to have a higher burden of baseline CAD, suggests that vulnerable plaque is the predominant feature in the pathophysiology of Ischemic heart disease (IHD). We propose that this may be due to a chronic inflammatory state, even in those who are virally suppressed. The mechanism for this finding and the effect of statins in this group needs evaluation.

\section{Acknowledgements The authors would like to acknowledge the Australian Heart Foundation.}

Funding CJH has received research funding and consultancy fees from Gilead Sciences. AC has received research funding from Bristol-Myers Squibb, Gilead Sciences, MSD and ViiV Healthcare; consultancy fees from Gilead Sciences, MSD and ViiV Healthcare; lecture and travel sponsorships from Bristol-Myers Squibb, Gilead Sciences, Janssen, MSD and ViiV Healthcare; and has served on advisory boards for Gilead Sciences, MSD and ViiV Healthcare.

Competing interests None declared.
Ethics approval Human Research and Ethics Committee (HREC) LNR/15/ $\mathrm{SVH} / 45$.

Provenance and peer review Not commissioned; externally peer reviewed.

Data sharing statement No additional data are available.

Open Access This is an Open Access article distributed in accordance with the Creative Commons Attribution Non Commercial (CC BY-NC 4.0) license, which permits others to distribute, remix, adapt, build upon this work noncommercially, and license their derivative works on different terms, provided the original work is properly cited and the use is non-commercial. See: http:// creativecommons.org/licenses/by-nc/4.0/

\section{REFERENCES}

1. Porter K, Babiker A, Bhaskaran K, et al. CASCADE Collaboration. Determinants of survival following HIV-1 seroconversion after the introduction of HAART. Lancet 2003;362:1267-74.

2. Boccara F, Lang S, Meuleman C, et al. HIV and coronary heart disease: time for a better understanding. J Am Coll Cardiol 2013;61:511-23.

3. Mathers CD, Loncar D. Projections of global mortality and burden of disease from 2002 to 2030. PLoS Med 2006;3:e442.

4. Smith CJ, Ryom L, Weber R, et al. D:A:D Study Group. Trends in underlying causes of death in people with HIV from 1999 to 2011 (D:A:D): a multicohort collaboration. Lancet 2014;384:241-8.

5. Lo J, Plutzky J. The biology of atherosclerosis: general paradigms and distinct pathogenic mechanisms among HIV-infected patients. $J$ Infect Dis 2012;205(Suppl 3):S368-74.

6. Becker AC, Sliwa K, Stewart S, et al. Acute coronary syndromes in treatment-naïve black South Africans with human immunodeficiency virus infection. J Interv Cardiol 2010;23:70-7.

7. Becker AC, Jacobson B, Singh S, et al. The thrombotic profile of treatment-naive HIV-positive Black South Africans with acute coronary syndromes. Clin Appl Thromb Hemost 2011;17:264-72.

8. Lo J, Abbara S, Shturman L, et al. Increased prevalence of subclinical coronary atherosclerosis detected by coronary computed tomography angiography in HIV-infected men. AIDS 2010;24:243-53.

9. Zanni MV, Abbara S, Lo J, et al. Increased coronary atherosclerotic plaque vulnerability by coronary computed tomography angiography in HIV-infected men. AIDS 2013;27:1263-72.

10. Post WS, Budoff M, Kingsley L, et al. Associations between HIV infection and subclinical coronary atherosclerosis. Ann Intern Med 2014;160:458-67.

11. Hsue PY, Giri K, Erickson S, et al. Clinical features of acute coronary syndromes in patients with human immunodeficiency virus infection. Circulation 2004;109:316-19.

12. Gensini GG. A more meaningful scoring system for determining the severity of coronary heart disease. Am J Cardiol 1983;51:606.

13. Triant VA, Lee $\mathrm{H}$, Hadigan $\mathrm{C}$, et al. Increased acute myocardial infarction rates and cardiovascular risk factors among patients with human immunodeficiency virus disease. J Clin Endocrinol Metab 2007;92:2506-12.

14. Friis-Moller N, Sabin CA, Weber R, et al. Data collection on adverse events of anti-HIV drugs (DAD) Study Group. Combination antiretroviral therapy and the risk of myocardial infarction. $N$ Engl J Med 2003;349:1993-2003.

15. Calza L, Vanino E, Salvadori C, et al. Tenofovir/emtricitabine/ efavirenz plus rosuvastatin decrease serum levels of inflammatory markers more than antiretroviral drugs alone in antiretroviral therapy-naive HIV-infected patients. HIV Clin Trials 2014;15:1-13.

16. Lo J, Lu MT, Ihenachor EJ, et al. Effects of statin therapy on coronary artery plaque volume and high-risk plaque morphology in HIV-infected patients with subclinical atherosclerosis: a randomised, double-blind, placebo-controlled trial. Lancet HIV 2015;2:e52-63.

17. El-Sadr WM, Lundgren J, Neaton JD, Strategies for Management of Antiretroviral Therapy (SMART) Study Group. CD4+count-guided interruption of antiretroviral treatment. N Engl J Med 2006;355:2283-96.

18. Borges ÁH, Weitz Jl, Gary Collins, et al., INSIGHT SILCAAT Scientific Committee. Markers of inflammation and activation of coagulation are associated with anemia in antiretroviral-treated HIV disease. AIDS 2014;28:1791-6.

19. Lundgren JD, Babiker AG, Gordin F, The INSIGHT START Study Group. Initiation of antiretroviral therapy in early asymptomatic HIV infection. N Engl J Med 2015;373:795-807.

20. Longenecker CT, Sullivan C, Baker JV. Immune activation and cardiovascular disease in chronic HIV infection. Curr Opin HIV AIDS 2016;11:216-25. 
21. Kelly KM, Tocchetti CG, Lyashkov A, et al. CCR5 inhibition prevents cardiac dysfunction in the SIV/macaque model of HIV. J Am Heart Assoc 2014;3:e000874.

22. Niccoli G, Stefanini GG, Capodanno D, et al. Are the Culprit Lesions Severely Stenotic? JACC Cardiovasc Imaging 2013;6:1108-14.
23. Glagov S, Weisenberg E, Zarins CK, et al. Compensatory enlargement of human atherosclerotic coronary arteries. N Engl $J$ Med 1987;316:1371-5.

24. Neeland IJ, Patel RS, Eshtehardi P, et al. Coronary angiographic scoring systems: an evaluation of their equivalence and validity. Am Heart J 2012;164:547-52. 